\title{
Effects of Various Antihypertensive Drugs on Arterial Stiffness and Wave Reflections
}

\author{
Ming Liu Ge-Le Li Yan Li Ji-Guang Wang \\ Centre for Epidemiological Studies and Clinical Trials, The Shanghai Institute of \\ Hypertension, Ruijin Hospital, Shanghai Jiaotong University School of Medicine, \\ Shanghai, China
}

\section{Key Words}

Antihypertensive drugs · Arterial stiffness - Wave reflections - Randomized controlled trial

\begin{abstract}
We reviewed trials that tested the efficacy of antihypertensive drugs in reducing arterial stiffness and wave reflections as assessed by pulse wave velocity and augmentation index, respectively. Regardless of cross-over or parallel-group comparison design, placebo-controlled trials demonstrated that antihypertensive drugs were effective in reducing pulse wave velocity. In actively-controlled parallel-group comparison studies, this effect on arterial stiffness was more evident for angiotensin-converting enzyme inhibitors or angiotensin receptor blockers than other classes of antihypertensive drugs, particularly when brachial-ankle pulse wave velocity was measured. Regardless of cross-over or parallel-group comparison or placebo- or activelycontrolled design, the reviewed trials showed that $\beta$-blockers were inferior to all the other classes of antihypertensive drugs in reducing augmentation index. However, these studies had a small sample size and a short follow-up time and did not link the changes in measurements of arterial function with cardiovascular events. Whether the superiority or inferiority is clinically relevant for cardiovascular protection and prevention remains to be investigated.
\end{abstract}

(c) 2013 S. Karger AG, Basel

\section{Introduction}

In the past 2 decades, noninvasive measurements of arterial function are increasingly used as an intermediate measure of cardiovascular disease risk in therapeutic trials, such as antihypertensive therapy. Among various parameters of arterial function, pulse wave velocity and augmentation index measure arterial stiffness and wave reflections, respectively. Both 
Liu et al.: Effects of Various Antihypertensive Drugs on Arterial Stiffness and Wave Reflections

measures can be accurately estimated within minutes with easy-to-use devices and may predict cardiovascular events above and beyond conventional cardiovascular risk factors, such as high blood pressure $[1,2]$. However, at present, there is no specific treatment for increased arterial stiffness or wave reflections. Nonetheless, antihypertensive drugs, especially those of vasodilatating action, seem to be promising in this regard.

Since the early 1990s, several randomized controlled trials have been conducted to study the effects of various antihypertensive drugs on carotid-femoral or brachial-ankle pulse wave velocity and augmentation index. In the present review article, we summarized these trials to investigate whether and which antihypertensive drugs are efficacious in reducing arterial stiffness and wave reflections and to explore the clinical relevance of these arterial measurements for cardiovascular protection and prevention.

\section{Arterial Effects of Antihypertensive Drugs in Placebo-Controlled Trials}

Of the 27 placebo-controlled trials, 11 had a cross-over design (table 1) [3-13] and 16 had a parallel-group comparison design (table 2) [14-29]. Regardless of the design, these placebo-controlled studies had a sample size of tens and a follow-up time of weeks.

Of the 11 placebo-controlled cross-over trials, 6 and 5 had single [8-13] and multiple comparisons with placebo [3-7], respectively, and 2, 4, and 5 studied pulse wave velocity [11, $13]$, augmentation index alone $[4,5,7,10]$, and both $[3,6,8,9,12]$, respectively (table 1$)$. The results of these trials were generally consistent across various classes of antihypertensive drugs for pulse wave velocity but not augmentation index. Antihypertensive drugs were efficacious in reducing pulse wave velocity. However, most drugs had neutral effects on augmentation index, and $\beta$-blockers even had worse effects than placebo on this measure of wave reflections $[6,12]$.

Of the 16 placebo-controlled parallel-group comparison trials, 12 and 4 had single [15, 17-22, 24, 25, 27-29] and multiple comparisons with placebo [14, 16, 23, 26], respectively, and 8,3 , and 5 studied pulse wave velocity $[14,16,19,24-28]$, augmentation index alone [15, $18,23]$, and both [17, 20-22, 29], respectively (table 2). The results of these parallel-group comparison trials were confirmatory for pulse wave velocity. Antihypertensive drugs significantly reduced pulse wave velocity in 10 of 18 drug comparisons from 13 trials. However, the results of these trials were slightly different for augmentation index. Antihypertensive drugs significantly reduced pulse wave velocity in 4 of 10 drug comparisons from 8 trials, none of which used $\beta$-blockers.

\section{Arterial Effects of Antihypertensive Drugs in Actively-Controlled Trials}

The actively-controlled trials also included those studies involving 2 or more drug comparisons with placebo. Of the 15 trials with a cross-over design (table 3) [1-5, 10, 30-38] and 31 trials with a parallel-group comparison design (table 4) [13, 16, 23, 26, 39-65], 6 [1-5, $10]$ and $4[13,16,23,26]$, respectively, were part of placebo-controlled studies. These activelycontrolled studies also had a small sample size and short follow-up time with the exception of the CAFE $(n=2,073)$ [61], EXPLORE $(n=331)$ [50], and REASON trials $(n=406)$ [65]. These bigger studies investigated combination therapy and (except REASON [65]) had an open design, and hence had limited information on the comparison between drug classes.

Of the cross-over trials, 2,7 , and 6 studied pulse wave velocity [31,37], augmentation index alone [2, 3, 5, 32, 33, 36, 38], and both $[1,4,10,30,34,35]$, respectively (table 3). These trials included 15 comparisons of angiotensin-converting enzyme (ACE) inhibitors with 
Liu et al.: Effects of Various Antihypertensive Drugs on Arterial Stiffness and Wave Reflections

Table 1. Randomized placebo-controlled double-blind cross-over studies

\begin{tabular}{|c|c|c|c|c|c|c|}
\hline \multirow{2}{*}{$\begin{array}{l}\text { First author } \\
\text { [Ref.] }\end{array}$} & \multirow[t]{2}{*}{ Year } & \multirow[t]{2}{*}{ Subjects } & \multirow{2}{*}{$\begin{array}{l}\text { Patients, } \\
\mathrm{n}\end{array}$} & \multirow{2}{*}{$\begin{array}{l}\text { Antihypertensive } \\
\text { treatment(s) }\end{array}$} & \multicolumn{2}{|l|}{ Results } \\
\hline & & & & & arterial stiffness & wave reflections \\
\hline \multicolumn{7}{|l|}{ ACEIs } \\
\hline Pannier [3] & 2001 & $\mathrm{EH}$ & 20 & perindopril & AUC cfPWV NS & AUC AIx perindopril better \\
\hline Deary [4] & 2002 & $\mathrm{EH}$ & 30 & lisinopril & not measured & AIx NS \\
\hline Morgan [5] & 2004 & $\mathrm{EH}$ & 32 & ACEIs & not measured & AIx NS \\
\hline Hirata [6] & 2005 & CAD & 30 & ramipril & cfPWV ramipril better & $\begin{array}{l}\text { AIx and AIx@HR75 } \\
\text { ramipril better }\end{array}$ \\
\hline Turner [7] & 2006 & $\begin{array}{l}\text { intracranial } \\
\text { aneurysms }\end{array}$ & 19 & perindopril & not measured & AIx NS \\
\hline \multicolumn{7}{|l|}{$A R B s$} \\
\hline Asmar [8] & 2002 & EH/DM & 20 & telmisartan & cfPWV telmisartan better & AIx NS \\
\hline Rajagopalan [9] & 2006 & healthy volunteers & 33 & valsartan & cfPWV NS & AIx NS \\
\hline Turner [7] & 2006 & $\begin{array}{l}\text { intracranial } \\
\text { aneurysms }\end{array}$ & 19 & irbesartan & not measured & AIx NS \\
\hline Kaufman [10] & 2010 & $\mathrm{EH}$ & 10 & losartan & not measured & AIx NS \\
\hline \multicolumn{7}{|l|}{$\beta$-Blockers } \\
\hline Asmar [11] & 1991 & $\mathrm{EH}$ & 14 & bisoprolol & cfPWV bisoprolol better & not measured \\
\hline Pannier [3] & 2001 & $\mathrm{EH}$ & 20 & atenolol & AUC cfPWV atenolol better & AUC AIx NS \\
\hline Deary [4] & 2002 & $\mathrm{EH}$ & 30 & bisoprolol & not measured & AIx bisoprolol better \\
\hline Morgan [5] & 2004 & $\mathrm{EH}$ & 32 & $\beta$-blockers & not measured & AIx NS \\
\hline Hirata [6] & 2005 & CAD & 30 & atenolol & cfPWV atenolol better & $\begin{array}{l}\text { AIx atenolol worse; } \\
\text { AIx@HR75 NS }\end{array}$ \\
\hline Dhakam [12] & 2008 & $\mathrm{EH}$ & 16 & nebivolol & aPWV nebivolol better & AIx nebivolol worse \\
\hline & & & & atenolol & aPWV atenolol better & AIx atenolol worse \\
\hline \multicolumn{7}{|l|}{ CCBs } \\
\hline Deary [4] & 2002 & $\mathrm{EH}$ & 30 & amlodipine & not measured & AIx NS \\
\hline Morgan [5] & 2004 & $\mathrm{EH}$ & 32 & CCBs & not measured & AIx NS \\
\hline \multicolumn{7}{|l|}{ Diuretics } \\
\hline Deary [4] & 2002 & $\mathrm{EH}$ & 30 & bendrofluazide & not measured & AIx NS \\
\hline Morgan [5] & 2004 & $\mathrm{EH}$ & 32 & diuretics & not measured & AIx NS \\
\hline Davies [13] & 2005 & $\mathrm{EH} / \mathrm{DM}$ & 10 & spironolactone & crPWV spironolactone better & not measured \\
\hline
\end{tabular}

ACEIs = ACE inhibitors; AIx = augmentation index; AIx@HR75 = AIx corrected for heart rate of 75 beats $/ \mathrm{min}$; aPWV = aortic pulse wave velocity; $\mathrm{AUC}=$ area under the curve; $\mathrm{CAD}=$ coronary artery disease; $\mathrm{cfPWV}=$ carotid-femoral pulse wave velocity; $\mathrm{crPWV}=$ carotid-radial pulse wave velocity; DM = diabetes mellitus; $\mathrm{EH}$ = essential hypertension; NS = not significantly different.

angiotensin receptor blockers (ARBs; $n=3)[5,30,31], \beta$-blockers $(n=7)[1-4,32-34]$, calcium channel blockers (CCBs; $n=3)[2,3,33]$, and diuretics $(n=2)[2,3], 2$ comparisons of ARBs with $\beta$-blockers [35, 36], 6 comparisons between 2 different $\beta$-blockers $(n=1)$ [10] or of $\beta$-blockers with CCBs $(n=3)[2,3,33]$ and diuretics $(n=2)[2,3], 3$ comparisons of CCBs with diuretics $[2,3,37]$, and 2 comparisons of combination therapy with each of their component drugs $[30,38]$. In these short-term cross-over studies, antihypertensive drugs had similar arterial effects, except that $\beta$-blockers were inferior to the other classes of antihypertensive drugs in reducing augmentation index in 11 of 14 comparisons with ACE inhibitors, ARBs, CCBs, or diuretics.

Of the parallel group trials, 20, 4, and 7 studied pulse wave velocity [13, 16, 26, 39-43, $46,47,49,52-56,58-60,62]$, augmentation index alone [23, 48, 57, 63], and both [44, 45, 50, $51,61,64,65]$, respectively (table 4). These trials included 20 comparisons of ACE inhibitors with ARBs $(n=6)[16,39-43]$, $\beta$-blockers $(n=2)[13,44]$, CCBs $(n=8)[13,39,40,43-46]$, and diuretics $(n=4)$ [44, 47-49], 13 comparisons of ARBs with $\beta$-blockers $(n=2)[50,51]$, 
Liu et al.: Effects of Various Antihypertensive Drugs on Arterial Stiffness and Wave Reflections

Table 2. Randomized placebo-controlled parallel-group comparison studies

\begin{tabular}{|c|c|c|c|c|c|c|c|}
\hline \multirow{2}{*}{$\begin{array}{l}\text { First author } \\
\text { [Ref.] }\end{array}$} & \multirow[t]{2}{*}{ Year } & \multirow{2}{*}{$\begin{array}{l}\text { De- } \\
\text { sign }\end{array}$} & \multirow[t]{2}{*}{ Subjects } & \multirow{2}{*}{$\begin{array}{l}\text { Patients, } \\
\mathrm{n}\end{array}$} & \multirow{2}{*}{$\begin{array}{l}\text { Antihypertensive } \\
\text { treatment(s) }\end{array}$} & \multicolumn{2}{|l|}{ Results } \\
\hline & & & & & & arterial stiffness & wave reflections \\
\hline \multicolumn{8}{|l|}{ ACEIs } \\
\hline Kahonen [14] & 1998 & DB & healthy volunteers & 15 & captopril & cfPWV captopril better & not measured \\
\hline Dart [15] & 2001 & open & $\mathrm{EH}$ & 111 & perindopril & not measured & AIx NS \\
\hline Ichihara [16] & 2005 & - & hemodialysis patients & 42 & trandolapril & baPWV trandolapril better & not measured \\
\hline $\mathrm{Yu}[17]$ & 2006 & DB & hemodialysis patients & 46 & ramipril & cfPWV NS & AIx NS \\
\hline Tsang [18] & 2006 & DB & IDD & 21 & quinapril & not measured & AIx NS \\
\hline Ahimastos [19] & 2007 & DB & Marfan syndrome & 17 & perindopril & $\begin{array}{l}\text { cfPWV and faPWV } \\
\text { perindopril better }\end{array}$ & not measured \\
\hline \multirow[t]{2}{*}{ Rahman [20] } & 2007 & DB & $\mathrm{DM}$ & 21 & ramipril & cfPWV NS & AIx NS \\
\hline & & & IGT & 19 & ramipril & cfPWV NS & AIx ramipril better \\
\hline Mitchell [21] & 2007 & open & CAD & 300 & trandolapril & cfPWV trandolapril better & AIx NS \\
\hline Ahimastos [22] & 2008 & DB & PAD & 40 & ramipril & cfPWV ramipril better & AIx ramipril better \\
\hline \multicolumn{8}{|l|}{$A R B s$} \\
\hline Klingbeil [23] & 2002 & DB & $\mathrm{EH}$ & 40 & valsartan & not measured & AIx valsartan better \\
\hline Ichihara [16] & 2005 & - & hemodialysis patients & 43 & losartan & baPWV NS & not measured \\
\hline $\begin{array}{l}\text { Mitsuhashi } \\
{[24]}\end{array}$ & 2009 & - & $\begin{array}{l}\text { EH/hemodialysis } \\
\text { patients }\end{array}$ & 40 & losartan & baPWV NS & not measured \\
\hline \multicolumn{8}{|l|}{$\beta$-Blockers } \\
\hline Kahonen [14] & 1998 & DB & healthy volunteers & 15 & propranolol & cfPWV propranolol better & not measured \\
\hline Kahonen [25] & 2000 & DB & healthy volunteers & 31 & $\begin{array}{l}\text { bisoprolol, } \\
\text { celiprolol, and } \\
\text { propranolol }\end{array}$ & $\begin{array}{l}\text { cfPWV bisoprolol and } \\
\text { propranolol better; } \\
\text { celiprolol worse }\end{array}$ & not measured \\
\hline Ylitalo [26] & 2005 & DB & healthy volunteers & 18 & bisoprolol & cfPWV NS & not measured \\
\hline \multicolumn{8}{|l|}{$C C B s$} \\
\hline London [27] & 1990 & DB & ESRD & 37 & nitrendipine & cfPWV nitrendipine better & not measured \\
\hline Asmar [28] & 1992 & DB & $\mathrm{EH}$ & 17 & nitrendipine & cfPWV nitrendipine better & not measured \\
\hline Kahonen [14] & 1998 & DB & healthy volunteers & 15 & verapamil & cfPWV NS & not measured \\
\hline Ylitalo [26] & 2005 & DB & healthy volunteers & 17 & nisoldipine & cfPWV NS & not measured \\
\hline \multicolumn{8}{|l|}{ Diuretics } \\
\hline Klingbeil [23] & 2002 & DB & $\mathrm{EH}$ & 40 & $\begin{array}{l}\text { hydrochlorothi- } \\
\text { azide }\end{array}$ & not measured & AIx NS \\
\hline Edwards [29] & 2009 & DB & CKD & 112 & spironolactone & $\begin{array}{l}\text { cfPWV spironolactone } \\
\text { better }\end{array}$ & $\begin{array}{l}\text { AIx spironolactone } \\
\text { better }\end{array}$ \\
\hline
\end{tabular}

ACEIs = ACE inhibitors; AIx = augmentation index; baPWV = brachial-ankle pulse wave velocity; CAD = coronary artery disease; $\mathrm{cfPWV}=$ carotid-femoral pulse wave velocity; $\mathrm{CKD}=$ chronic kidney disease; $\mathrm{DB}=$ double-blinded; $\mathrm{DM}=$ diabetes mellitus; $\mathrm{EH}=$ essential hypertension; ESRD = end-stage renal dysfunction; faPWV = femoral-dorsalis pedis pulse wave velocity; IDD = isolated diastolic dysfunction; IGT = impaired glucose tolerance; NS = not significantly different; PAD = peripheral artery disease.

CCBs $(n=10)[39,40,43,52-58]$, or a diuretic $(n=1)$ [23], 5 comparisons of $\beta$-blockers with CCBs $(n=4)[13,26,44,59]$ or a diuretic $(n=1)[44], 7$ comparisons between 2 different CCBs $(n=1)[40]$ or of CCBs with diuretics $(n=6)[44,60-64]$, and 2 comparisons of combination therapy with one [65] or two of their component drugs [41]. In these studies, ACE inhibitors or ARBs tended to be more efficacious than other classes of antihypertensive drugs in reducing arterial stiffness, especially when brachial-ankle pulse wave velocity was measured in 11 trials $[16,40,41,43,46,52-54,56,58,62]$. The results were not consistent for other comparisons of pulse wave velocity or for studies on augmentation index, except that $\beta$-blockers were inferior to the other classes of antihypertensive drugs in reducing augmentation index in all 5 comparisons with an ACE inhibitor $(n=1)$ [44], ARBs $(n=2)[50,51]$, a CCB $(n=1)$ [44], or a diuretic $(n=1)$ [44]. 
Liu et al.: Effects of Various Antihypertensive Drugs on Arterial Stiffness and Wave Reflections

Table 3. Randomized actively-controlled cross-over studies

\begin{tabular}{|c|c|c|c|c|c|c|c|}
\hline \multirow{2}{*}{$\begin{array}{l}\text { First author } \\
\text { [Ref.] }\end{array}$} & \multirow[t]{2}{*}{ Year } & \multirow{2}{*}{$\begin{array}{l}\text { De- } \\
\text { sign }\end{array}$} & \multirow[t]{2}{*}{ Subjects } & \multirow{2}{*}{$\begin{array}{l}\text { Patients, } \\
\mathrm{n}\end{array}$} & \multirow{2}{*}{ Comparison(s) } & \multicolumn{2}{|l|}{ Results } \\
\hline & & & & & & arterial stiffness & wave reflections \\
\hline \multicolumn{8}{|l|}{ ACEIs } \\
\hline Mahmud [30] & 2002 & SB & $\mathrm{EH}$ & 12 & captopril vs. valsartan & cfPWV NS & AIx NS \\
\hline Turner [7] & 2006 & DB & $\begin{array}{l}\text { intracranial } \\
\text { aneurysms }\end{array}$ & 19 & perindopril vs. irbesartan & not measured & AIx NS \\
\hline Ali [31] & 2009 & DB & $\mathrm{EH}$ & 15 & lisinopril vs. irbesartan & cfPWV NS & not measured \\
\hline \multicolumn{8}{|l|}{ vs. $\beta$-blockers } \\
\hline Chen [32] & 1995 & DB & $\mathrm{EH}$ & 79 & fosinopril vs. atenolol & not measured & $\begin{array}{l}\text { Alx fosinopril } \\
\text { better }\end{array}$ \\
\hline Pannier [3] & 2001 & DB & $\mathrm{EH}$ & 20 & perindopril vs. atenolol & $\begin{array}{l}\text { cfPWV atenolol } \\
\text { better }\end{array}$ & $\begin{array}{l}\text { AUC AIx peri- } \\
\text { dopril better }\end{array}$ \\
\hline Deary [4] & 2002 & DB & $\mathrm{EH}$ & 30 & lisinopril vs. bisoprolol & not measured & $\begin{array}{l}\text { AIx lisinopril } \\
\text { better }\end{array}$ \\
\hline Morgan [5] & 2004 & DB & $\mathrm{EH}$ & 32 & ACEIs vs. $\beta$-blockers & not measured & AIx ACEIs better \\
\hline Neal [33] & 2004 & DB & $\begin{array}{l}\text { EH/liver } \\
\text { transplan- } \\
\text { tation }\end{array}$ & 12 & lisinopril vs. bisoprolol & not measured & $\begin{array}{l}\text { AIx lisinopril } \\
\text { better }\end{array}$ \\
\hline Hirata [6] & 2005 & DB & CAD & 30 & ramipril vs. atenolol & cfPWV NS & $\begin{array}{l}\text { AIx and } \\
\text { AIx@HR75 } \\
\text { ramipril better }\end{array}$ \\
\hline Kaiser [34] & 2006 & DB & $\mathrm{EH} / \mathrm{DM}$ & 10 & enalapril vs. nebivolol & cfPWV NS & AIx NS \\
\hline \multicolumn{8}{|l|}{ vs. CCBs } \\
\hline Deary [4] & 2002 & DB & $\mathrm{EH}$ & 30 & lisinopril vs. amlodipine & not measured & AIx NS \\
\hline Morgan [5] & 2004 & DB & $\mathrm{EH}$ & 32 & ACEIs vs. CCBs & not measured & AIx NS \\
\hline Neal [33] & 2004 & DB & $\begin{array}{l}\text { EH/liver } \\
\text { transplan- } \\
\text { tation }\end{array}$ & 12 & lisinopril vs. amlodipine & not measured & AIx NS \\
\hline \multicolumn{8}{|l|}{ vs. diuretics } \\
\hline Deary [4] & 2002 & DB & EH & 30 & lisinopril vs. bendrofluazide & not measured & AIx NS \\
\hline Morgan [5] & 2004 & DB & $\mathrm{EH}$ & 32 & ACEIs vs. diuretics & not measured & AIx NS \\
\hline \multicolumn{8}{|l|}{ ARBs } \\
\hline \multicolumn{8}{|l|}{ vs. $\beta$-blockers } \\
\hline Dhakam [35] & 2006 & DB & $\mathrm{EH}$ & 21 & eprosartan vs. atenolol & $\begin{array}{l}\text { cfPWV atenolol } \\
\text { better }\end{array}$ & $\begin{array}{l}\text { AIx eprosartan } \\
\text { better }\end{array}$ \\
\hline Izzo [36] & 2012 & SB & $\mathrm{EH}$ & 30 & $\begin{array}{l}\text { lisinopril + valsartan vs. } \\
\text { lisinopril + carvedilol }\end{array}$ & not measured & AIx NS \\
\hline \multicolumn{8}{|l|}{$\beta$-Blockers } \\
\hline Dhakam [12] & 2008 & DB & $\mathrm{EH}$ & 16 & atenolol vs. nebivolol & aPWV NS & $\begin{array}{l}\text { AIx nebivolol } \\
\text { better }\end{array}$ \\
\hline \multicolumn{8}{|l|}{ vs. $C C B s$} \\
\hline Deary [4] & 2002 & DB & $\mathrm{EH}$ & 30 & bisoprolol vs. amlodipine & not measured & $\begin{array}{l}\text { AIx amlodipine } \\
\text { better }\end{array}$ \\
\hline Morgan [5] & 2004 & DB & $\mathrm{EH}$ & 32 & $\beta$-blockers vs. CCBs & not measured & AIx CCBs better \\
\hline Neal [33] & 2004 & DB & $\begin{array}{l}\text { EH/liver } \\
\text { transplan- } \\
\text { tation }\end{array}$ & 12 & bisoprolol vs. amlodipine & not measured & AIx NS \\
\hline \multicolumn{8}{|l|}{ vs. diuretics } \\
\hline Deary [4] & 2002 & DB & $\mathrm{EH}$ & 30 & bisoprolol vs. bendrofluazide & not measured & $\begin{array}{l}\text { Alx bendro- } \\
\text { fluazide better }\end{array}$ \\
\hline Morgan [5] & 2004 & $\mathrm{DB}$ & $\mathrm{EH}$ & 32 & $\beta$-blockers vs. diuretics & not measured & $\begin{array}{l}\text { Alx diuretics } \\
\text { better }\end{array}$ \\
\hline
\end{tabular}


Liu et al.: Effects of Various Antihypertensive Drugs on Arterial Stiffness and Wave Reflections

Table 3 (continued)

\begin{tabular}{|c|c|c|c|c|c|c|c|}
\hline \multirow{2}{*}{$\begin{array}{l}\text { First author } \\
\text { [Ref.] }\end{array}$} & \multirow[t]{2}{*}{ Year } & \multirow{2}{*}{$\begin{array}{l}\text { De- } \\
\text { sign }\end{array}$} & \multirow[t]{2}{*}{ Subjects } & \multirow{2}{*}{$\begin{array}{l}\text { Patients, } \\
\mathrm{n}\end{array}$} & \multirow{2}{*}{ Comparison(s) } & \multicolumn{2}{|l|}{ Results } \\
\hline & & & & & & arterial stiffness & wave reflections \\
\hline \multicolumn{8}{|l|}{ CCBs } \\
\hline \multicolumn{8}{|l|}{ vs. diuretics } \\
\hline Asmar [37] & 1993 & DB & $\mathrm{EH}$ & 16 & $\begin{array}{l}\text { felodipine vs. hydrochloro- } \\
\text { thiazide }\end{array}$ & $\begin{array}{l}\text { cfPWV felodipine } \\
\text { better }\end{array}$ & not measured \\
\hline Deary [4] & 2002 & DB & $\mathrm{EH}$ & 30 & amlodipine vs. bendrofluazide & not measured & AIx NS \\
\hline Morgan [5] & 2004 & DB & $\mathrm{EH}$ & 32 & CCBs vs. diuretics & not measured & AIx NS \\
\hline \multicolumn{8}{|l|}{ Other } \\
\hline Mahmud [30] & 2002 & SB & EH & 12 & $\begin{array}{l}\text { captopril + valsartan vs. } \\
\text { captopril } \\
\text { vs. valsartan }\end{array}$ & $\begin{array}{l}\text { cfPWV } \\
\text { combination } \\
\text { better }\end{array}$ & $\begin{array}{l}\text { AIx combination } \\
\text { better }\end{array}$ \\
\hline Ferguson [38] & 2008 & DB & EH & 22 & $\begin{array}{l}\text { fosinopril + hydrochloro- } \\
\text { thiazide vs. amlodipine } \\
\text { vs. indapamide }\end{array}$ & not measured & $\begin{array}{l}\text { Alx combination } \\
\text { better }\end{array}$ \\
\hline
\end{tabular}

ACEIs = ACE inhibitors; AIx = augmentation index; AIx@HR75 = AIx corrected for heart rate of 75 beats $/$ min; aPWV = aortic pulse wave velocity; $\mathrm{AUC}=$ area under the curve; $\mathrm{CAD}=$ coronary artery disease; $\mathrm{cfPWV}=$ carotid-femoral pulse wave velocity; $\mathrm{DB}=$ doubleblinded; DM = diabetes mellitus; $\mathrm{EH}$ = essential hypertension; NS = not significantly different; SB = single-blinded.

\section{Conclusions and Perspectives}

Our narrative review was informative on three clinically relevant questions. First, antihypertensive drugs are effective in reducing arterial stiffness. However, this effect does not at all infer any benefit above and beyond blood pressure lowering. In contrast, because pulse wave velocity is dependent on systolic blood pressure, the therapeutic effect of antihypertensive drugs on arterial stiffness, to some extent if not entirely, can be attributable to their blood pressure lowering efficacy. Second, though all antihypertensive drugs reduce arterial stiffness, ACE inhibitors or ARBs might be more efficacious than other classes of antihypertensive drugs. This effect is more evident when brachial-ankle pulse wave velocity is measured. The mechanism remains to be elucidated. Third, as also evidenced by a recent meta-analysis of randomized controlled trials that compared $\beta$-blockers with the other classes of antihypertensive drugs [66], because of the intrinsic heart rate slowing effect, $\beta$-blockers are inferior to all the other classes of antihypertensive drugs in reducing augmentation index. However, whether this inferiority is clinically relevant for cardiovascular protection and prevention remains to be investigated.

In spite of a large number of trials that studied the efficacy of antihypertensive drugs in reducing pulse wave velocity and augmentation index, these studies had a small sample size and a short follow-up time and did not link the changes in measurements of arterial function with cardiovascular events. It is therefore imperative to combine the research force in the field of arterial functions to run adequately powered outcome trials to investigate whether arterial stiffness and wave reflections are clinically useful in monitoring the effect of antihypertensive treatment and other cardiovascular therapeutic approaches. 
Liu et al.: Effects of Various Antihypertensive Drugs on Arterial Stiffness and Wave

Reflections

Table 4. Randomized actively-controlled parallel-group comparison studies

\begin{tabular}{|c|c|c|c|c|c|c|c|}
\hline \multirow[t]{2}{*}{ First author } & \multirow[t]{2}{*}{ Year } & \multirow{2}{*}{$\begin{array}{l}\text { De- } \\
\text { sign }\end{array}$} & \multirow[t]{2}{*}{ Subjects } & \multirow{2}{*}{$\begin{array}{l}\text { Patients, } \\
\mathrm{n}\end{array}$} & \multirow[t]{2}{*}{ Comparison(s) } & \multicolumn{2}{|l|}{ Results } \\
\hline & & & & & & arterial stiffness & wave reflections \\
\hline \multicolumn{8}{|l|}{ ACEIs } \\
\hline \multicolumn{8}{|l|}{ vs. $A R B s$} \\
\hline Rajzer [39] & 2003 & open & $\mathrm{EH}$ & 62 & quinapril vs. losartan & cfPWV quinapril better & not measured \\
\hline Takami [40] & 2003 & SB & $\mathrm{EH}$ & 40 & temocapril vs. valsartan & baPWV valsartan better & not measured \\
\hline Ichihara [16] & 2005 & SB & $\begin{array}{l}\text { hemodialysis } \\
\text { patients }\end{array}$ & 43 & trandolapril vs. losartan & baPWV NS & not measured \\
\hline Anan [41] & 2005 & SB & $\mathrm{EH}$ & 21 & perindopril vs. valsartan & baPWV NS & not measured \\
\hline Rehman [42] & 2007 & DB & $\mathrm{EH}$ & 39 & perindopril vs. losartan & cfPWV NS & not measured \\
\hline $\operatorname{Li}[43]$ & 2009 & $\mathrm{SB}$ & $\mathrm{EH}$ & 68 & perindopril vs. telmisartan & $\begin{array}{l}\text { baPWV telmisartan } \\
\text { better }\end{array}$ & not measured \\
\hline \multicolumn{8}{|l|}{ vs. $\beta$-blockers } \\
\hline Kahonen [14] & 1998 & DB & $\begin{array}{l}\text { healthy } \\
\text { volunteers }\end{array}$ & 15 & captopril vs. propranolol & aPWV NS & not measured \\
\hline Mackenzie [44] & 2009 & DB & $\mathrm{EH}$ & 32 & perindopril vs. atenolol & cfPWV NS & $\begin{array}{l}\text { AIx perindopril } \\
\text { better }\end{array}$ \\
\hline \multicolumn{8}{|l|}{ vs. $C C B s$} \\
\hline London [45] & 1994 & SB & ESRD & 24 & perindopril vs. nitrendipine & cfPWV NS & AIx NS \\
\hline Kahonen [14] & 1998 & DB & $\begin{array}{l}\text { healthy } \\
\text { volunteers }\end{array}$ & 15 & captopril vs. verapamil & aPWV NS & not measured \\
\hline Rajzer [39] & 2003 & open & $\mathrm{EH}$ & 75 & quinapril vs. amlodipine & cfPWV quinapril better & not measured \\
\hline \multirow[t]{2}{*}{ Takami [40] } & 2003 & - & $\mathrm{EH}$ & 40 & temocapril vs. cilnidipine & baPWV NS & not measured \\
\hline & & & & 36 & temocapril vs. nifedipine & $\begin{array}{l}\text { baPWV temocapril } \\
\text { better }\end{array}$ & not measured \\
\hline Morimoto [46] & 2008 & - & $\mathrm{EH}$ & 32 & $\begin{array}{l}\text { ARB + perindopril vs. } \\
\text { ARB + amlodipine }\end{array}$ & baPWV NS & not measured \\
\hline Mackenzie [44] & 2009 & DB & $\mathrm{EH}$ & 29 & perindopril vs. lercanidipine & cfPWV NS & AIx NS \\
\hline $\operatorname{Li}[43]$ & 2009 & SB & $\mathrm{EH}$ & 68 & perindopril vs. amlodipine & $\begin{array}{l}\text { baPWV amlodipine } \\
\text { better }\end{array}$ & not measured \\
\hline \multicolumn{8}{|l|}{ vs. diuretics } \\
\hline $\begin{array}{l}\text { Breithaupt- } \\
\text { Grogler [47] }\end{array}$ & 1996 & $\mathrm{DB}$ & $\mathrm{EH}$ & 17 & $\begin{array}{l}\text { cilazapril vs. } \\
\text { hydrochlorothiazide }\end{array}$ & cfPWV NS & not measured \\
\hline Jiang [48] & 2005 & DB & $\mathrm{EH}$ & 101 & enalapril vs. indapamide & not measured & AIx enalapril better \\
\hline Mackenzie [44] & 2009 & DB & $\mathrm{EH}$ & 28 & perindopril vs. bendrofluazide & cfPWV NS & AIx NS \\
\hline $\begin{array}{l}\text { Kostka-Jeziorny } \\
\text { [49] }\end{array}$ & 2011 & open & $\mathrm{EH}$ & 66 & $\begin{array}{l}\text { perindopril vs. } \\
\text { hydrochlorothiazide }\end{array}$ & cfPWV NS & not measured \\
\hline \multicolumn{8}{|l|}{ ARBs } \\
\hline \multicolumn{8}{|l|}{ vs. $\beta$-blockers } \\
\hline Boutouyrie [50] & 2010 & open & $\mathrm{EH}$ & 331 & $\begin{array}{l}\text { amlodipine + valsartan vs. } \\
\text { amlodipine + atenolol }\end{array}$ & cfPWV NS & $\begin{array}{l}\text { AIx and AIx@HR75 } \\
\text { valsartan better }\end{array}$ \\
\hline Vitale $[51]$ & 2012 & DB & $\mathrm{EH}$ & 65 & irbesartan vs. nebivolol & cfPWV NS & $\begin{array}{l}\text { AIx irbesartan better; } \\
\text { AIx@HR75 NS }\end{array}$ \\
\hline \multicolumn{8}{|l|}{ vs. $C C B s$} \\
\hline Rajzer [39] & 2003 & open & $\mathrm{EH}$ & 61 & losartan vs. amlodipine & cfPWV NS & not measured \\
\hline Takami [40] & 2003 & - & $\mathrm{EH}$ & 40 & $\begin{array}{l}\text { valsartan vs. cilnidipine vs. } \\
\text { nifedipine }\end{array}$ & baPWV valsartan better & not measured \\
\hline Munakata [52] & 2004 & - & $\mathrm{EH}$ & 41 & valsartan vs. nifedipine & baPWV valsartan better & not measured \\
\hline Ichihara [53] & 2006 & - & $\mathrm{EH}$ & 100 & valsartan vs. amlodipine & baPWV NS & not measured \\
\hline Morimoto [54] & 2006 & - & $\mathrm{EH}$ & 43 & telmisartan vs. amlodipine & $\begin{array}{l}\text { baPWV telmisartan } \\
\text { better }\end{array}$ & not measured \\
\hline Kosch [55] & 2008 & $\mathrm{DB}$ & $\mathrm{EH}$ & 52 & valsartan vs. metoprolol & cfPWV NS & not measured \\
\hline Ishii [56] & 2008 & - & $\mathrm{EH} / \mathrm{DM}$ & 22 & candesartan vs. CCBs & $\begin{array}{l}\text { baPWV candesartan } \\
\text { better }\end{array}$ & not measured \\
\hline Schneider [57] & 2008 & DB & $\mathrm{EH}$ & 156 & irbesartan vs. atenolol & not measured & Alx irbesartan better \\
\hline $\operatorname{Li}[43]$ & 2009 & $\mathrm{SB}$ & $\mathrm{EH}$ & 68 & telmisartan vs. amlodipine & $\begin{array}{l}\text { baPWV telmisartan } \\
\text { better }\end{array}$ & not measured \\
\hline Tomiyama [58] & 2011 & - & $\mathrm{EH}$ & 113 & candesartan vs. amlodipine & $\begin{array}{l}\text { baPWV candesartan } \\
\text { better }\end{array}$ & not measured \\
\hline
\end{tabular}


Liu et al.: Effects of Various Antihypertensive Drugs on Arterial Stiffness and Wave

Table 4 (continued)

\begin{tabular}{|c|c|c|c|c|c|c|c|}
\hline \multirow[t]{2}{*}{ First author } & \multirow[t]{2}{*}{ Year } & \multirow{2}{*}{$\begin{array}{l}\text { De- } \\
\text { sign }\end{array}$} & \multirow[t]{2}{*}{ Subjects } & \multirow{2}{*}{$\begin{array}{l}\text { Patients, } \\
\mathrm{n}\end{array}$} & \multirow{2}{*}{ Comparison(s) } & \multicolumn{2}{|l|}{ Results } \\
\hline & & & & & & arterial stiffness & wave reflections \\
\hline \multicolumn{8}{|l|}{ vs. diuretics } \\
\hline Klingbeil [23] & 2002 & DB & $\mathrm{EH}$ & 40 & $\begin{array}{l}\text { valsartan vs. } \\
\text { hydrochlorothiazide }\end{array}$ & not measured & AIx valsartan better \\
\hline \multicolumn{8}{|l|}{$\beta$-Blockers } \\
\hline \multicolumn{8}{|l|}{ vs. CCBs } \\
\hline Merli [59] & 1993 & DB & $\mathrm{EH}$ & 28 & metoprolol vs. isradipine & cfPWV isradipine better & not measured \\
\hline Kahonen [14] & 1998 & DB & $\begin{array}{l}\text { healthy } \\
\text { volunteers }\end{array}$ & 15 & propranolol vs. verapamil & $\begin{array}{l}\text { cfPWV propranolol } \\
\text { better }\end{array}$ & not measured \\
\hline Ylitalo [26] & 2005 & DB & $\begin{array}{l}\text { healthy } \\
\text { volunteers }\end{array}$ & 18 & bisoprolol vs. nisoldipine & cfPWV NS & not measured \\
\hline Mackenzie [44] & 2009 & DB & $\mathrm{EH}$ & 31 & atenolol vs. lercanidipine & cfPWV NS & $\begin{array}{l}\text { AIx lercanidipine } \\
\text { better }\end{array}$ \\
\hline \multicolumn{8}{|l|}{ vs. diuretics } \\
\hline Mackenzie [44] & 2009 & DB & $\mathrm{EH}$ & 30 & atenolol vs. bendrofluazide & cfPWV NS & $\begin{array}{l}\text { AIx bendrofluazide } \\
\text { better }\end{array}$ \\
\hline \multicolumn{8}{|l|}{ CCBs } \\
\hline \multicolumn{8}{|l|}{ vs. $C C B s$} \\
\hline Takami [40] & 2003 & - & $\mathrm{EH}$ & 36 & cilnidipine vs. nifedipine & $\begin{array}{l}\text { baPWV cilnidipine } \\
\text { better }\end{array}$ & not measured \\
\hline \multicolumn{8}{|l|}{ vs. diuretics } \\
\hline White [60] & 2003 & $\mathrm{DB}$ & $\mathrm{EH}$ & 139 & amlodipine vs. eplerenone & cfPWV NS & not measured \\
\hline Williams [61] & 2006 & open & $\mathrm{EH}$ & 2,073 & amlodipine vs. atenolol & cfPWV NS (n = 114) & $\begin{array}{l}\text { Alx amlodipine } \\
\text { better }\end{array}$ \\
\hline Kaneshiro [62] & 2009 & DB & CKD & 68 & $\begin{array}{l}\text { valsartan + amlodipine vs. } \\
\text { valsartan + thiazide }\end{array}$ & baPWV NS & not measured \\
\hline Mackenzie [44] & 2009 & DB & $\mathrm{EH}$ & 27 & $\begin{array}{l}\text { lercanidipine vs. } \\
\text { bendrofluazide }\end{array}$ & cfPWV NS & AIx NS \\
\hline Doi [63] & 2010 & open & $\mathrm{EH}$ & 37 & $\begin{array}{l}\text { azelnidipine vs. } \\
\text { trichlormethiazide }\end{array}$ & not measured & $\begin{array}{l}\text { AIx and AIx@HR75 } \\
\text { azelnidipine better }\end{array}$ \\
\hline Matsui [64] & 2011 & open & $\mathrm{EH}$ & 207 & $\begin{array}{l}\text { azelnidipine vs. } \\
\text { hydrochlorothiazide }\end{array}$ & $\begin{array}{l}\text { cfPWV azelnidipine } \\
\text { better }\end{array}$ & AIx NS \\
\hline \multicolumn{8}{|l|}{ Other } \\
\hline Asmar [65] & 2001 & DB & $\mathrm{EH}$ & 406 & $\begin{array}{l}\text { perindopril + indapamide vs. } \\
\text { atenolol }\end{array}$ & cfPWV NS & $\begin{array}{l}\text { AIx combination } \\
\text { better }\end{array}$ \\
\hline Anan [41] & 2005 & - & $\mathrm{EH}$ & 21 & $\begin{array}{l}\text { perindopril + valsartan vs. } \\
\text { perindopril vs. valsartan }\end{array}$ & $\begin{array}{l}\text { baPWV combination } \\
\text { better }\end{array}$ & not measured \\
\hline $\begin{array}{l}\text { ACEIs = ACE } \\
\text { wave velocity; ba } \\
\text { DB = double-blin } \\
\text { different; SB = si }\end{array}$ & $\begin{array}{l}\text { inhibitc } \\
\text { PWV = } \\
\text { ded; DI } \\
\text { ngle-bli }\end{array}$ & $\begin{array}{l}\text { rs; AIx } \\
\text { prachia } \\
\text { M = dial } \\
\text { nded. }\end{array}$ & $\begin{array}{l}=\text { augmentat } \\
\text { l-ankle pulse } \\
\text { betes mellitu }\end{array}$ & $\begin{array}{l}\text { on index } \\
\text { wave velc } \\
; \mathrm{EH}=\mathrm{es}\end{array}$ & $\begin{array}{l}\text {; AIx@HR75 = AIx corrected fo } \\
\text { ocity; } \text { cfPWV = carotid-femoral } \\
\text { sential hypertension; ESRD = }\end{array}$ & $\begin{array}{l}\text { heart rate of } 75 \text { beats } / \mathrm{mi} \\
\text { ulse wave velocity; } C K D=c \\
\text { d-stage renal dysfunction }\end{array}$ & $\begin{array}{l}\text { n; aPWV = aortic pulse } \\
\text { hronic kidney disease } \\
\text {; NS = not significantly }\end{array}$ \\
\hline
\end{tabular}

\section{Acknowledgement}

The authors were financially supported by grants from the National Natural Science Foundation of China (grants 30871360, 30871081, 81170245, and 81270373), the Ministry of Science and Technology (a grant for China-European Union collaborations, 1012), and the Ministry of Education (NCET-09-0544), Beijing, China, the Shanghai Commissions of Science and Technology (grant 07JC14047, the 'Rising Star' program 06QA14043, and 11QH1402000) and Education (grant 07ZZ32 and the 'Dawn' project 08SG20), the Shanghai Bureau of Health (XBR2011004), Shanghai Jiaotong University School of Medicine (a grant of Distinguished Young Investigators to Y.L.), and the European Union (grants LSHM-CT-2006-037093 and HEALTH-F4-2007-201550). 
Liu et al.: Effects of Various Antihypertensive Drugs on Arterial Stiffness and Wave Reflections

\section{Disclosure Statement}

J.-G.W. reports receiving lecture and consulting fees from A\&D, Boehringer-Ingelheim, MSD, Novartis, Omron, Pfizer, Servier, and Takeda.

\section{References}

1 Vlachopoulos C, Aznaouridis K, Stefanadis C: Prediction of cardiovascular events and all-cause mortality with arterial stiffness: a systematic review and meta-analysis. J Am Coll Cardiol 2010;55:1318-1327.

2 Vlachopoulos C, Aznaouridis K, O’Rourke MF, Safar ME, Baou K, Stefanadis C: Prediction of cardiovascular events and all-cause mortality with central haemodynamics: a systematic review and meta-analysis. Eur Heart J 2010;31:1865-1871.

3 Pannier BM, Guerin AP, Marchais SJ, London GM: Different aortic reflection wave responses following longterm angiotensin-converting enzyme inhibition and beta-blocker in essential hypertension. Clin Exp Pharmacol Physiol 2001;28:1074-1077.

- 4 Deary AJ, Schumann AL, Murfet H, Haydock S, Foo RS, Brown MJ: Influence of drugs and gender on the arterial pulse wave and natriuretic peptide secretion in untreated patients with essential hypertension. Clin Sci (Lond) 2002;103:493-499.

5 Morgan T, Lauri J, Bertram D, Anderson A: Effect of different antihypertensive drug classes on central aortic pressure. Am J Hypertens 2004;17:118-123.

- 6 Hirata K, Vlachopoulos C, Adji A, O’Rourke MF: Benefits from angiotensin-converting enzyme inhibitor 'beyond blood pressure lowering': beyond blood pressure or beyond the brachial artery? J Hypertens 2005;23:551556.

7 Turner CL, Wilkinson IB, Kirkpatrick PJ: Use of antihypertension agents for the suppression of arterial pulse pressure waveforms in patients with intracranial aneurysms. J Neurosurg 2006;104:531-536.

- 8 Asmar R, Gosse P, Topouchian J, N’Tela G, Dudley A, Shepherd GL: Effects of telmisartan on arterial stiffness in type 2 diabetes patients with essential hypertension. J Renin Angiotensin Aldosterone Syst 2002;3:176-180.

- 9 Rajagopalan S, Kariisa M, Dellegrottaglie S, Bard RL, Kehrer C, Matlow S, Daley W, Pitt B, Brook R: Angiotensin receptor blockade improves vascular compliance in healthy normotensive elderly individuals: results from a randomized double-blind placebo-controlled trial. J Clin Hypertens (Greenwich) 2006;8:783-790.

10 Kaufman R, Nunes I, Bolognese JA, Miller DL, Salotti D, McCarthy JM, Smith WB, Herman GA, Feig PU: Singledose effects of isosorbidemononitrate alone or in combination with losartan on central blood pressure. J Am Soc Hypertens 2010;4:311-318.

11 Asmar RG, Kerihuel JC, Girerd XJ, Safar ME: Effect of bisoprolol on blood pressure and arterial hemodynamics in systemic hypertension. Am J Cardiol 1991;68:61-64.

-12 Dhakam Z, Yasmin, McEniery CM, Burton T, Brown MJ, Wilkinson IB: A comparison of atenolol and nebivolol in isolated systolic hypertension. J Hypertens 2008;26:351-356.

13 Davies J, Gavin A, Band M, Morris A, Struthers A: Spironolactone reduces brachial pulse wave velocity and PIIINP levels in hypertensive diabetic patients. Br J Clin Pharmacol 2005;59:520-523.

14 Kahonen M, Ylitalo R, Koobi T, Turjanmaa V, Ylitalo P: Influence of captopril, propranolol, and verapamil on arterial pulse wave velocity and other cardiovascular parameters in healthy volunteers. Int J Clin Pharmacol Ther 1998;36:483-489.

15 Dart AM, Reid CM, McGrath B: Effects of ACE inhibitor therapy on derived central arterial waveforms in hypertension. Am J Hypertens 2001;14:804-810.

-16 Ichihara A, Hayashi M, Kaneshiro Y, Takemitsu T, Homma K, Kanno Y, Yoshizawa M, Furukawa T, Takenaka T, Saruta T: Low doses of losartan and trandolapril improve arterial stiffness in hemodialysis patients. Am J Kidney Dis 2005;45:866-874.

17 Yu WC, Lin YP, Lin IF, Chuang SY, Chen CH: Effect of ramipril on left ventricular mass in normotensive hemodialysis patients. Am J Kidney Dis 2006;47:478-484.

18 Tsang TS, Barnes ME, Abhayaratna WP, Cha SS, Gersh BJ, Langins AP, Green TD, Bailey KR, Miyasaka Y, Seward JB: Effects of quinapril on left atrial structural remodeling and arterial stiffness. Am J Cardiol 2006;97:916920.

19 Ahimastos AA, Aggarwal A, D’Orsa KM, Formosa MF, White AJ, Savarirayan R, Dart AM, Kingwell BA: Effect of perindopril on large artery stiffness and aortic root diameter in patients with Marfan syndrome: a randomized controlled trial. JAMA 2007;298:1539-1547.

20 Rahman S, Ismail AA, Ismail SB, Naing NN, Abdul RA: Effect of rosiglitazone/ramipril on preclinical vasculopathy in newly diagnosed, untreated diabetes and IGT patients: 1-year randomised, double-blind, placebocontrolled study. Eur J Clin Pharmacol 2007;63:733-741.

21 Mitchell GF, Dunlap ME, Warnica W, Ducharme A, Arnold JM, Tardif JC, Solomon SD, Domanski MJ, Jablonski KA, Rice MM, Pfeffer MA: Long-term trandolapril treatment is associated with reduced aortic stiffness: the prevention of events with angiotensin-converting enzyme inhibition hemodynamic substudy. Hypertension 2007;49:1271-1277. 
Liu et al.: Effects of Various Antihypertensive Drugs on Arterial Stiffness and Wave Reflections

22 Ahimastos AA, Dart AM, Lawler A, Blombery PA, Kingwell BA: Reduced arterial stiffness may contribute to angiotensin-converting enzyme inhibitor induced improvements in walking time in peripheral arterial disease patients. J Hypertens 2008;26:1037-1042.

-23 Klingbeil AU, John S, Schneider MP, Jacobi J, Weidinger G, Schmieder RE: AT1-receptor blockade improves augmentation index: a double-blind, randomized, controlled study. J Hypertens 2002;20:2423-2428.

-24 Mitsuhashi H, Tamura K, Yamauchi J, Ozawa M, Yanagi M, Dejima T, Wakui H, Masuda S, Azuma K, Kanaoka T, Ohsawa M, Maeda A, Tsurumi-Ikeya Y, Okano Y, Ishigami T, Toya Y, Tokita Y, Ohnishi T, Umemura S: Effect of losartan on ambulatory short-term blood pressure variability and cardiovascular remodeling in hypertensive patients on hemodialysis. Atherosclerosis 2009;207:186-190.

25 Kahonen M, Ylitalo R, Koobi T, Turjanmaa V, Ylitalo P: Influences of nonselective, beta(1)-selective and vasodilatory beta(1)-selective beta-blockers on arterial pulse wave velocity in normotensive subjects. Gen Pharmacol 2000;35:219-224.

26 Ylitalo R, Kahonen M, Nieminen T, Koobi T, Ylitalo P, Turjanmaa V: Effects of a mononitrate, a beta1-blocker and a dihydropyridine calcium channel blocker on cardiovascular responsiveness to passive orthostasis: a placebo-controlled double-blind study in normotensive volunteers. Arzneimittelforschung 2005;55:160-166.

27 London GM, Marchais SJ, Guerin AP, Metivier F, Safar ME, Fabiani F, Froment L: Salt and water retention and calcium blockade in uremia. Circulation 1990;82:105-113.

28 Asmar R, Benetos A, Brahimi M, Chaouche K, Safar M: Arterial and antihypertensive effects of nitrendipine: a double-blind comparison versus placebo. J Cardiovasc Pharmacol 1992;20:858-863.

-29 Edwards NC, Steeds RP, Stewart PM, Ferro CJ, Townend JN: Effect of spironolactone on left ventricular mass and aortic stiffness in early-stage chronic kidney disease: a randomized controlled trial. J Am Coll Cardiol 2009;54:505-512.

-30 Mahmud A, Feely J: Reduction in arterial stiffness with angiotensin II antagonist is comparable with and additive to ACE inhibition. Am J Hypertens 2002;15:321-325.

-31 Ali K, Rajkumar C, Fantin F, Schiff R, Bulpitt CJ: Irbesartan improves arterial compliance more than lisinopril. Vasc Health Risk Manag 2009;5:587-592.

-32 Chen CH, Ting CT, Lin SJ, Hsu TL, Yin FC, Siu CO, Chou P, Wang SP, Chang MS: Different effects of fosinopril and atenolol on wave reflections in hypertensive patients. Hypertension 1995;25:1034-1041.

-33 Neal DA, Brown MJ, Wilkinson IB, Byrne CD, Alexander GJ: Hemodynamic effects of amlodipine, bisoprolol, and lisinopril in hypertensive patients after liver transplantation. Transplantation 2004;77:748-750.

34 Kaiser T, Heise T, Nosek L, Eckers U, Sawicki PT: Influence of nebivolol and enalapril on metabolic parameters and arterial stiffness in hypertensive type 2 diabetic patients. J Hypertens 2006;24:1397-1403.

-35 Dhakam Z, McEniery CM, Yasmin, Cockcroft JR, Brown MJ, Wilkinson IB: Atenolol and eprosartan: differential effects on central blood pressure and aortic pulse wave velocity. Am J Hypertens 2006;19:214-219.

-36 Izzo JJ, Rajpal M, Karan S, Srikakarlapudi S, Osmond PJ: Hemodynamic and central blood pressure differences between carvedilol and valsartan added to lisinopril at rest and during exercise stress. J Am Soc Hypertens 2012;6:117-123.

37 Asmar RG, Benetos A, Chaouche-Teyara K, Raveau-Landon CM, Safar ME: Comparison of effects of felodipine versus hydrochlorothiazide on arterial diameter and pulse-wave velocity in essential hypertension. Am J Cardiol 1993;72:794-798.

38 Ferguson JM, Minas J, Siapantas S, Komesaroff PA, Sudhir K: Effects of a fixed-dose ACE inhibitor-diuretic combination on ambulatory blood pressure and arterial properties in isolated systolic hypertension. J Cardiovasc Pharmacol 2008;51:590-595.

-39 Rajzer M, Klocek M, Kawecka-Jaszcz K: Effect of amlodipine, quinapril, and losartan on pulse wave velocity and plasma collagen markers in patients with mild-to-moderate arterial hypertension. Am J Hypertens 2003;16: 439-444.

-40 Takami T, Shigemasa M: Efficacy of various antihypertensive agents as evaluated by indices of vascular stiffness in elderly hypertensive patients. Hypertens Res 2003;26:609-614.

-41 Anan F, Takahashi N, Ooie T, Yufu K, Hara M, Nakagawa M, Yonemochi H, Saikawa T, Yoshimatsu H: Effects of valsartan and perindopril combination therapy on left ventricular hypertrophy and aortic arterial stiffness in patients with essential hypertension. Eur J Clin Pharmacol 2005;61:353-359.

-42 Rehman A, Ismail SB, Naing L, Roshan TM, Rahman AR: Reduction in arterial stiffness with angiotensin II antagonism and converting enzyme inhibition. A comparative study among Malay hypertensive subjects with a known genetic profile. Am J Hypertens 2007;20:184-189.

-43 Li Y, Ma SM, Du M, Chu WW, Cheng XM: Perindopril, amlodipine and telmisartan improve arterial stiffness in patients with hypertension (in Chinese). Zhonghua Xin Xue Guan Bing Za Zhi 2009;37:908-912.

-44 Mackenzie IS, McEniery CM, Dhakam Z, Brown MJ, Cockcroft JR, Wilkinson IB: Comparison of the effects of antihypertensive agents on central blood pressure and arterial stiffness in isolated systolic hypertension. Hypertension 2009;54:409-413.

45 London GM, Pannier B, Guerin AP, Marchais SJ, Safar ME, Cuche JL: Cardiac hypertrophy, aortic compliance, peripheral resistance, and wave reflection in end-stage renal disease. Comparative effects of ACE inhibition and calcium channel blockade. Circulation 1994;90:2786-2796.

46 Morimoto S, Maki K, Aota Y, Sakuma T, Iwasaka T: Beneficial effects of combination therapy with angiotensin II receptor blocker and angiotensin-converting enzyme inhibitor on vascular endothelial function. Hypertens Res 2008;31:1603-1610. 
Liu et al.: Effects of Various Antihypertensive Drugs on Arterial Stiffness and Wave Reflections

-47 Breithaupt-Grogler K, Leschinger M, Belz GG, Butzer R, Erb K, de May C, Sinn W: Influence of antihypertensive therapy with cilazapril and hydrochlorothiazide on the stiffness of the aorta. Cardiovasc Drugs Ther 1996;10: 49-57.

-48 Jiang XJ, Li QY, Zhang YQ, Liu GZ, Liu LS: The comparison of the effect of enalapril and indapamide on the peripheral blood pressure and central blood pressure through pulse wave analysis (in Chinese). Zhonghua Xin Xue Guan Bing Za Zhi 2005;33:885-888.

49 Kostka-Jeziorny K, Uruski P, Tykarski A: Effect of allopurinol on blood pressure and aortic compliance in hypertensive patients. Blood Press 2011;20:104-110.

-50 Boutouyrie P, Achouba A, Trunet P, Laurent S: Amlodipine-valsartan combination decreases central systolic blood pressure more effectively than the amlodipine-atenolol combination: the EXPLOR study. Hypertension 2010;55:1314-1322.

51 Vitale C, Marazzi G, Iellamo F, Spoletini I, Dall'Armi V, Fini M, Volterrani M: Effects of nebivolol or irbesartan in combination with hydrochlorothiazide on vascular functions in newly-diagnosed hypertensive patients: the NINFE (Nebivololo, Irbesartan Nella Funzione Endoteliale) study. Int J Cardiol 2012;155:279-284.

52 Munakata M, Nagasaki A, Nunokawa T, Sakuma T, Kato H, Yoshinaga K, Toyota T: Effects of valsartan and nifedipine coat-core on systemic arterial stiffness in hypertensive patients. Am J Hypertens 2004;17:1050-1055.

-53 Ichihara A, Kaneshiro Y, Takemitsu T, Sakoda M: Effects of amlodipine and valsartan on vascular damage and ambulatory blood pressure in untreated hypertensive patients. J Hum Hypertens 2006;20:787-794.

54 Morimoto S, Yano Y, Maki K, Sawada K: Renal and vascular protective effects of telmisartan in patients with essential hypertension. Hypertens Res 2006;29:567-572.

55 Kosch M, Levers A, Lang D, Bartels V, Rahn KH, Pavenstadt H, Hausberg M: A randomized, double-blind study of valsartan versus metoprolol on arterial distensibility and endothelial function in essential hypertension. Nephrol Dial Transplant 2008;23:2280-2285.

-56 Ishii H, Tsukada T, Yoshida M: Angiotensin II type-I receptor blocker, candesartan, improves brachial-ankle pulse wave velocity independent of its blood pressure lowering effects in type 2 diabetes patients. Intern Med 2008;47:2013-2018.

57 Schneider MP, Delles C, Klingbeil AU, Ludwig M, Kolloch RE, Krekler M, Stumpe KO, Schmieder RE: Effect of angiotensin receptor blockade on central haemodynamics in essential hypertension: results of a randomised trial. J Renin Angiotensin Aldosterone Syst 2008;9:49-56.

58 Tomiyama H, Yoshida M, Yamada J, Matsumoto C, Odaira M, Shiina K, Yamashina A: Arterial-cardiac destiffening following long-term antihypertensive treatment. Am J Hypertens 2011;24:1080-1086.

59 Merli I, Simon A, Del PM, Brautigam M, Welzel D, Levenson J: Intrinsic effect of antihypertensive treatment with isradipine and metoprololon large artery geometric and elastic properties. Clin Pharmacol Ther 1993; 54:76-83.

-60 White WB, Duprez D, St Hillaire R, Krause S, Roniker B, Kuse-Hamilton J, Weber MA: Effects of the selective aldosterone blocker eplerenone versus the calcium antagonist amlodipine in systolic hypertension. Hypertension 2003;41:1021-1026.

-61 Williams B, Lacy PS, Thom SM, Cruickshank K, Stanton A, Collier D, Hughes AD, Thurston H, O’Rourke M: Differential impact of blood pressure-lowering drugs on central aortic pressure and clinical outcomes: principal results of the Conduit Artery Function Evaluation (CAFE) study. Circulation 2006;113:1213-1225.

62 Kaneshiro Y, Ichihara A, Sakoda M, Kurauchi-Mito A, Kinouchi K, Itoh H: Add-on benefits of amlodipine and thiazide in nondiabetic chronic kidney disease stage $1 / 2$ patients treated with valsartan. Kidney Blood Press Res 2009;32:51-58.

63 Doi M, Miyoshi T, Hirohata S, Kamikawa S, Usui S, Kaji Y, Sakane K, Ogawa H, Ninomiya Y, Kusachi S: Combination therapy of calcium channel blocker and angiotensin II receptor blocker reduces augmentation index in hypertensive patients. Am J Med Sci 2010;339:433-439.

64 Matsui Y, Eguchi K, O’Rourke MF, Ishikawa J, Shimada K, Kario K: Association between aldosterone induced by antihypertensive medication and arterial stiffness reduction: the J-CORE study. Atherosclerosis 2011;215: 184-188.

65 Asmar RG, London GM, O’Rourke ME, Safar ME: Improvement in blood pressure, arterial stiffness and wave reflections with a very-low-dose perindopril/indapamide combination in hypertensive patient: a comparison with atenolol. Hypertension 2001;38:922-926.

66 Ding FH, Li Y, Li LH, Wang JG: Impact of heart rate on central hemodynamics and stroke: a meta-analysis of $\beta$-blocker trials. Am J Hypertens 2013;26:118-125. 